\title{
Page curve from non-Markovianity
}

\author{
Kaixiang Su, ${ }^{a, b}$ Pengfei Zhang ${ }^{c}$ and Hui Zhai ${ }^{a}$ \\ ${ }^{a}$ Institute for Advanced Study, Tsinghua University, \\ 30 Shuangqing Road, Beijing 100084, China \\ ${ }^{b}$ Department of Physics, University of California Santa Barbara, \\ Broida Hall, Santa Barbara, California, 93106, U.S.A. \\ ${ }^{c}$ Institute of Quantum Information and Matter and Walter Burke Institute for Theoretical Physics, \\ California Institute of Technology, \\ 1200 E California Blvd, Pasadena, California 91125, U.S.A \\ E-mail: sukaixiang@pku.edu.cn, pengfeizhang.physics@gmail.com, \\ hzhai@tsinghua.edu.cn
}

ABSTRACT: In this paper, we use the exactly solvable Sachdev-Ye-Kitaev model to address the issue of entropy dynamics when an interacting quantum system is coupled to a nonMarkovian environment. We find that at the initial stage, the entropy always increases linearly matching the Markovian result. When the system thermalizes with the environment at a sufficiently long time, if the environment temperature is low and the coupling between system and environment is weak, then the total thermal entropy is low and the entanglement between system and environment is also weak, which yields a small system entropy in the long-time steady state. This manifestation of non-Markovian effects of the environment forces the entropy to decrease in the later stage, which yields the Page curve for the entropy dynamics. We argue that this physical scenario revealed by the exact solution of the Sachdev-Ye-Kitaev model is universally applicable for general chaotic quantum many-body systems and can be verified experimentally in near future.

KEywords: 1/N Expansion, Field Theories in Lower Dimensions, AdS-CFT Correspondence

ArXiv EPrint: 2101.11238 


\section{Contents}

1 Introduction 1

2 Model 2

3 Recovering the Markovian results 3

4 The Page curve $\quad 6$

$\begin{array}{lll}5 & \text { CFT bath and holography } & 7\end{array}$

6 Summary 8

A Path-integral representation of the Rényi entropy 9

A.1 Non-Markovian bath $\quad 9$

$\begin{array}{ll}\text { A.2 Markovian bath } & 11\end{array}$

B Perturbation results $\quad 12$

$\begin{array}{lll}\text { B.1 The short-time perturbation } & 12\end{array}$

$\begin{array}{lll}\text { B.2 Long time asymptotic behavior with small } \kappa & 13\end{array}$

$\begin{array}{ll}\text { C Numerical discretization } & 14\end{array}$

\section{Introduction}

Studying open quantum many-body systems is of fundamental importance for understanding quantum matters and for future applications of quantum technology because all systems are inevitably in contact with environments, and decoherence due to coupling with environments is a major obstacle for future applications of quantum devices [1]. So far, most studies of open quantum systems are limited to either situations in which the systems are small or weakly correlated, or situations that the environment is treated by the Born-Markovian approximation [2,3]. Little effort has been made on strongly correlated quantum many-body systems coupled to a non-Markovian environment. This is simply because both strong correlation and non-Markovianity are difficult to handle theoretically.

Open systems are also of interest to gravity studies, and the best-known problem is the black hole information paradox [4]. The central issue of the black hole information paradox is whether the black hole evaporation can be considered as undergoing unitary dynamics. If so, the entropy should first increase and then decrease as the black hole evaporates. Such an entropy curve as shown in figure 1(a) is known as the Page curve [5]. Here the entanglement entropy is the entropy of the reduced density matrix of the radiation part $\mathcal{A}$ after tracing out the remaining black hole part $\mathcal{B}$. As shown in figure $1(\mathrm{a})$, this entanglement entropy reaches the maximum when half of the black hole is evaporated, giving rise to the Page curve. Reproducing the Page curve from gravity theory is a challenging part of the black hole information problem, and progresses have been made recently using the semi-classical gravity calculations [6-10]. 
(a)
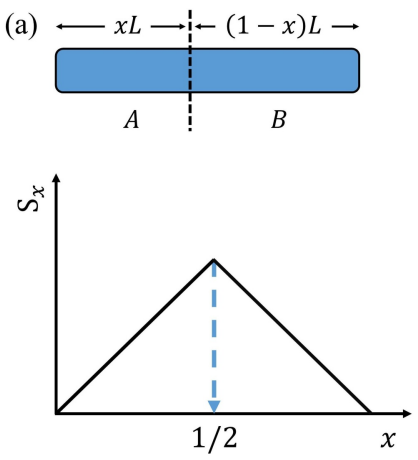

(b)

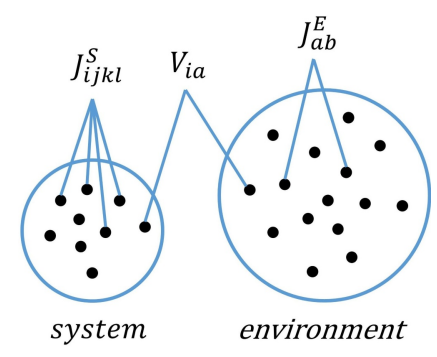

Figure 1. (a) Illustration of the Page curve: the entanglement entropy between two sub-systems with length $x L$ and $(1-x) L$. The total length is $L$ and $0 \leqslant x \leqslant 1$. (b) Illustration of the setup: an $\mathrm{SYK}_{4}$ system with $N$ Majorana fermions serves as the system and an $\mathrm{SYK}_{2}$ system with $M$ Majorana fermions serves as the environment. Here $M \gg N$.

In this paper, we explore the Sachdev-Ye-Kitaev model $[11,12]$ with random fourMajorana fermions interactions $\left(\mathrm{SYK}_{4}\right)$, and this $\mathrm{SYK}_{4}$ model is coupled to a system with random quadratic Majorana fermions couplings $\left(\mathrm{SYK}_{2}\right)$. The $\mathrm{SYK}_{2}$ part contains a lot more degrees-of-freedom compared with the $\mathrm{SYK}_{4}$ part such that the $\mathrm{SYK}_{2}$ part can be viewed as the environment. The motivations for considering such a model are two folds. First, the $\mathrm{SYK}_{4}$ model is exactly solvable in the large-N limit and its solution gives rise to a strongly correlated non-Fermi liquid state [11]. Recently, techniques related to $\mathrm{SYK}_{4}$ model has been widely used to construct exact solutions to address open issues of strongly correlated quantum matters [13-31]. Here the situation we explored is also exactly solvable and we can use the solution to understand how a non-Markovian environment affects strongly correlated phases [23-25]. Secondly, the SYK 4 model is holographically dual to the Jackiw-Teitelboim gravity theory in the $\mathrm{AdS}_{2}$ geometry with a black hole [32-37]. Thus, the entropy dynamics of the $\mathrm{SYK}_{4}$ system coupled to an environment $[22,26,31]$ resembles the black hole evaporation process [6-10] and it will be interesting to study when a Page-like curve can emerge after turning on the coupling between the system and the environment. Remarkably, the main findings of this work bring these two aspects together, that is, we show that the Page curve emerges because of the non-Markovian effect of the environment.

\section{Model}

The system under consideration is illustrated in figure 1(b) and the total Hamiltonian is given by

$$
\begin{aligned}
\hat{H} & =\hat{H}_{\mathrm{S}}+\hat{H}_{\mathrm{E}}+\hat{H}_{\mathrm{SE}} \\
& =\sum_{i<j<k<l} J_{i j k l}^{S} \psi_{i} \psi_{j} \psi_{k} \psi_{l}+i \sum_{a<b} J_{a b}^{\mathrm{E}} \chi_{a} \chi_{b}+i \sum_{i, a} V_{i a} \psi_{i} \chi_{a} .
\end{aligned}
$$

Here $\psi_{i}(i=1, \ldots, N)$ denotes $N$ Majorana fermions in the system and $\chi_{a}(a=1, \ldots, M)$ denotes $M$ Majorana fermions in the environment, with $\left\{\psi_{i}, \psi_{j}\right\}=\delta_{i j}$ and $\left\{\chi_{a}, \chi_{b}\right\}=\delta_{a b}$. 
Throughout the manuscript, we will use the subscript "S" and "E" to denote the system part and the environment part respectively. $\hat{H}_{\mathrm{S}}$ and $\hat{H}_{\mathrm{E}}$ are then $\mathrm{SYK}_{4}$ and $\mathrm{SYK}_{2}$ Hamiltonians. $J_{i j k l}^{S}, J_{a b}^{\mathrm{E}}$ and $V_{i a}$ are independent random Gaussian variables with variances given by

$$
\overline{\left(J_{i j k l}^{\mathrm{S}}\right)^{2}}=\frac{3 ! J_{S}^{2}}{N^{3}}, \quad \overline{\left(J_{a b}^{\mathrm{E}}\right)^{2}}=\frac{2 ! J_{\mathrm{E}}^{2}}{M}, \quad \overline{\left(V_{i a}\right)^{2}}=\frac{V^{2}}{M} .
$$

Throughout the paper, we take $J_{S}=1$ as the energy unit. We focus on the limit $M \gg N \gg 1$ in which the Schwinger-Dyson equation for $\chi$ contains no contribution from $\psi$, justifying that the $\chi$ part can be viewed as the environment. Therefore, the Green's function $G_{\chi}(\tau)=\left\langle T_{\tau} \chi_{i}(\tau) \chi_{i}(0)\right\rangle$ of the environment takes the standard form of the $\mathrm{SYK}_{2}$ model as [11]

$$
G_{\chi}(\omega)=-\frac{2}{i \omega+i \operatorname{sgn}(\omega) \sqrt{4 J_{\mathrm{E}}^{2}+\omega^{2}}} .
$$

The fact that this Green's function has frequency dependence means that the environment is treated beyond the Markovian approximation.

We consider the situation in which the system and the environment are initially decoupled, and both are in thermal equilibrium with inverse temperatures $\beta_{\mathrm{S}}$ and $\beta_{\mathrm{E}}$ respectively. The initial density matrix is therefore given by $\rho(0)=\frac{1}{Z_{\mathrm{S}} Z_{\mathrm{E}}} e^{-\beta_{S} \hat{H}_{\mathrm{S}}} \otimes e^{-\beta_{\mathrm{E}} \hat{H}_{\mathrm{E}}}$ with $Z_{\mathrm{S}}$ and $Z_{\mathrm{E}}$ being the corresponding partition functions. Evolving the system with the Hamiltonian eq. (2.1) and tracing out the environment, one obtains the reduced density matrix of the system $\rho_{\mathrm{S}}(t)$ at time $t$ as

$$
\rho_{S}(t)=\operatorname{tr}_{\mathrm{E}}\left[e^{-i \hat{H} t} \rho(0) e^{i \hat{H} t}\right] .
$$

The corresponding second Rényi entropy $S^{(2)}$ of the system is then given by

$$
e^{-S_{\mathrm{S}}^{(2)}(t)}=\operatorname{tr}_{\mathrm{S}}\left[\rho_{S}(t)^{2}\right]
$$

Under the disorder replica diagonal assumption, $S_{\mathrm{S}}^{(2)}(t)$ can be expressed in terms of pathintegral over bilocal fields. In the large- $N$ limit, the integral is dominated by the saddle point solution, and the entropy can be obtained by evaluating the on-shell action. We leave the details of the derivation into the appendix.

\section{Recovering the Markovian results}

Below we will first discuss situations where the entropy dynamics of our model can recover the Markovian results. Here, by Markovian results we mean dynamics obtained by solving the following Lindblad master equation $[2,3]$

$$
\partial_{t} \hat{\rho}=-i\left[\hat{H}_{\mathrm{S}}, \hat{\rho}\right]+\sum_{i} \kappa_{i}\left(\hat{L}_{i} \rho \hat{L}_{i}^{\dagger}-\frac{1}{2}\left\{\hat{L}_{i}^{\dagger} \hat{L}_{i}, \hat{\rho}\right\}\right)
$$

By treating the environment with the Markovian approximation, we only need to consider the Hamiltonian of the system and the dissipation operators, without having to explicitly include the environment. To make comparison with our model, we take $\kappa_{i}=\kappa$ and 
$\hat{L}_{i}=\psi_{i}$. Similar to previous procedures, we consider the initial thermal density matrix $\rho_{\mathrm{S}}(0)=\frac{1}{Z_{\mathrm{S}}} e^{-\beta_{\mathrm{S}} \hat{H}_{\mathrm{S}}}$, and we then evolve the system with eq. (3.1). The second Rényi entropy can also be represented as a path-integral where the saddle points approximation is applicable. In the Markovian case, the Rényi entropy first grows linearly in time and then saturates to its maximum value $(N / 2) \log 2$, forbidding the possibility of any page-like behaviors [38]. For reasons that will become clear below, we consider the large $J_{\mathrm{E}}$ limit and fix $V^{2} / J_{\mathrm{E}}$ as $\kappa$ in our model. Our discussions below will then identify the following conditions as sufficient for our model to recover the Markovian results.

(i) Infinite Environment Temperature. The $\mathrm{SYK}_{2}$ environment becomes a Markovian one when $\beta_{\mathrm{E}}=0$. This is because only the two-point function enters the effective action for entropy dynamics, and in the large $J_{\mathrm{E}}$ limit, the Fourier transformation of the real-time Green's function of the environment $G_{\mathrm{E}}^{>}(t, \beta)=\left\langle\chi_{a}(t) \chi_{a}(0)\right\rangle_{\beta} / Z_{B}$ gives

$$
G_{\mathrm{E}}^{>}\left(\omega, \beta_{\mathrm{E}}\right)=\frac{1}{J_{\mathrm{E}}} \frac{1}{1+e^{-\beta_{\mathrm{E}} \omega}} .
$$

When $\beta_{\mathrm{E}}=0$, the second term vanishes and the Green's function of the environment becomes frequency independent, which is equivalent to the Markovian approximation. Under this situation, the standard derivation of the master equation eq. (3.1) through a second-order perturbation theory yields the dissipation strength $\kappa=V^{2} / J_{\mathrm{E}}$. As one can see in figure $2(\mathrm{a})$, when $\beta_{\mathrm{E}}$ is set to zero, the entropy curve becomes independent of $\kappa$. This universal curve also coincides with the results from the Markovian approximation.

(ii) Short Time. With the Markovian approximation, it can be shown by the perturbation theory that the entropy grows linearly at the initial stage, and when $\kappa t \ll 1[38,39]$, the growth rate derived analytically in the appendix gives

$$
\frac{d S_{\mathrm{S}}^{(2)}(t)}{d t}=\kappa N\left(1-2 G_{\mathrm{S}}^{W}\left(0,2 \beta_{S}\right)\right) .
$$

Here we have defined the Wightman Green's function for the single $\mathrm{SYK}_{4}$ model as

$$
G_{\mathrm{S}}^{W}(t, \beta)=\frac{1}{Z_{\mathrm{S}}}\left\langle\psi_{i}\left(t-i \frac{\beta}{2}\right) \psi_{i}(0)\right\rangle_{\beta} .
$$

For our model, similar perturbative calculation [31] for short-time yields

$$
\begin{aligned}
\frac{d S_{\mathrm{S}}^{(2)}}{d t}=2 V^{2} N \int_{-t}^{t} d t^{\prime} & {\left[\left(G_{\mathrm{S}}^{>}\left(t^{\prime}, 2 \beta_{S}\right)-G_{\mathrm{S}}^{W}\left(t^{\prime}, 2 \beta_{S}\right)\right)\right.} \\
& \left.\times G_{\mathrm{E}}^{>}\left(t^{\prime}, \beta_{B}\right)\right] .
\end{aligned}
$$

By approximating $t^{\prime}=0$ in the integrand, eq. (3.5) becomes

$$
\frac{d S_{\mathrm{S}}^{(2)}}{d t}=\frac{V^{2}}{J_{\mathrm{E}}} N\left(1-2 G_{\mathrm{S}}^{W}\left(0,2 \beta_{S}\right)\right) .
$$




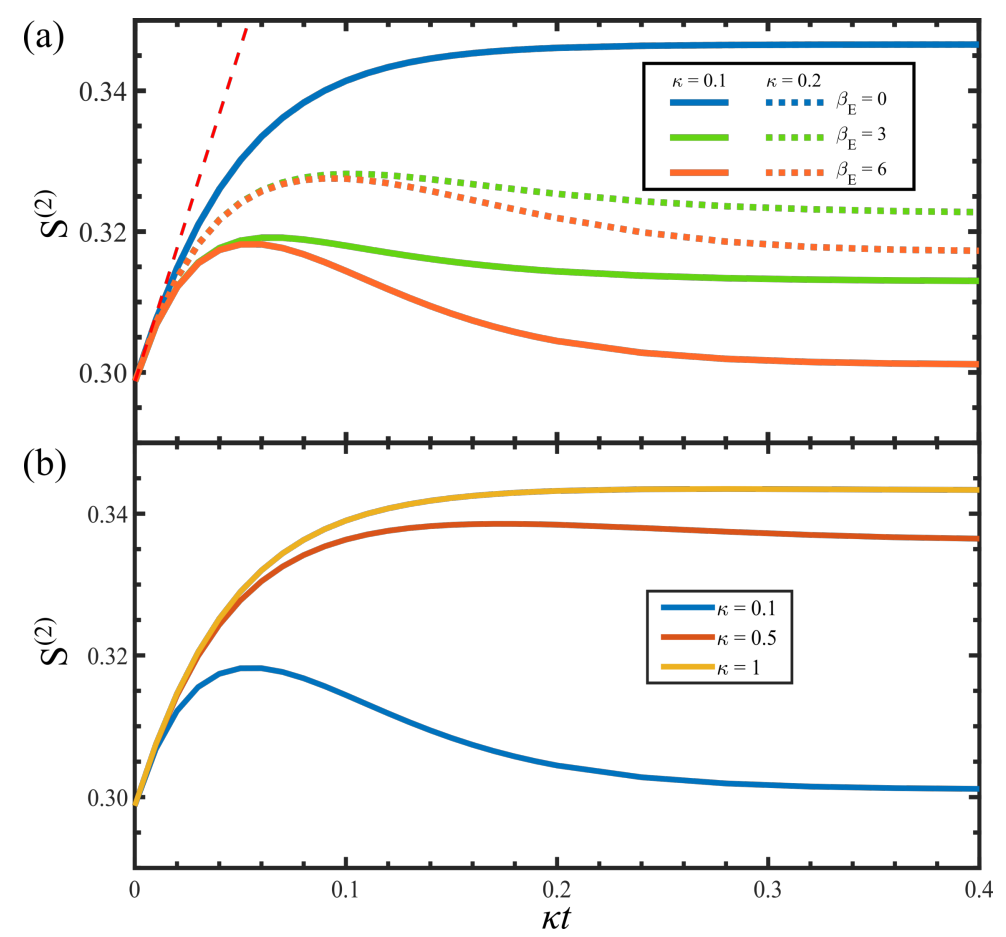

Figure 2. (a) Entropy curves for different $\kappa$ and $\beta_{\mathrm{E}}$. Here $\kappa=0.1$ for solid lines and $\kappa=0.2$ for dashed lines. Three different $\beta_{\mathrm{E}}=(0,3,6)$ are plotted. The red dashed straight line represents the same initial slope for all curves. Two curves with $\beta_{\mathrm{E}}=0$ in (a) coincide with the Markovian results. (b) Entropy curves with different $\kappa$ and a fixed $\beta_{\mathrm{E}}=6$. The curve with large $\kappa$ in (b) coincides with $\beta_{\mathrm{E}}=0$ curves in (a) and the Markovian results.

By equalling $V^{2} / J_{E}=\kappa$, eq. (3.6) is the same as eq. (3.3). This can also be seen in figure 2(a) that the initial growth is linear in $\kappa t$ and the slop is a constant for varying $\beta_{\mathrm{E}}$ with fixed $\beta_{\mathrm{S}}$. In other words, although the Green's function of the environment eq. (3.2) contains the frequency dependent part, it is not important for initial time and the short-time behavior is always dominated by the frequency independent part.

(iii) Large System-Environment Coupling. The entropy curve of our model also matches the Markovian results when $\kappa$ is sufficiently large compared to $J_{\mathrm{S}}$. Since the short time limit is always Markovian as discussed in ii), here we focus on the long-time limit. Physically, when the coupling between system and environment is strong enough compared with the internal energy scales of the system, all Majorana fermions in the system tend to be maximally entangled with the environment, because the environment contains more degrees of freedom. Consequently, the entropy is expected to saturate to the maximum value $(N / 2) \log 2$ in the long-time limit, which is the same as the Markovian case. This can also be shown more rigorously using the path-integral formalism by relating the Rényi entropy to the inner product of Kourkoulou-Maldacena pure states $[27,29]$. In figure $2(\mathrm{~b})$, one can see that the entropy curve gradually approaches the Markovian result as $\kappa$ increases. 


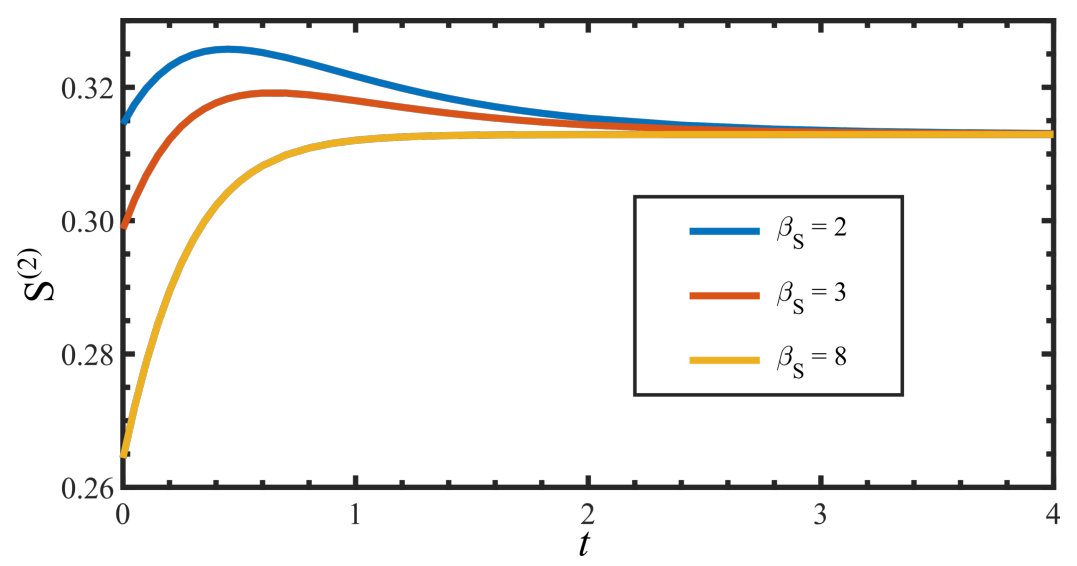

Figure 3. The entropy curve for varying $\beta_{\mathrm{S}}$ with fixed $\kappa=0.1$ and $\beta_{\mathrm{E}}=3$. Three different $\beta_{\mathrm{S}}=(2,3,8)$ are plotted. Page-like behaviours is guaranteed when $\beta_{\mathrm{S}}=2$ because the initial entropy is higher than the saturated entropy.

\section{The Page curve}

Above we have shown that, under these three situations, our model recovers the Markovian results, and the Markovian results do not display the Page curve for entropy dynamics. Hence, to reveal effects beyond the Markovian approximation, the following three conditions should be satisfied simultaneously, which are: i) the bath temperature should not be too high; ii) the evolution time should not be too short; iii) the coupling between system and environment should not be too large. Under these conditions, we find that Page curve for entropy dynamics is often observed, as was shown in figure 3. Thus, this attributes the emergence of the Page curve to the beyond Markovian effect. Since we have discussed that the entropy always increases at the initial time, it is essential to understand the decreasing behavior of the entropy curve at long time to understand the Page curve. Below we offer two physical understandings.

The first understanding again relies on perturbation theory. When $\kappa$ is small, the entropy dynamics can be obtained by doing perturbation in $\kappa$, which yields the same perturbative results as eq. (3.5). Here, since we focus on the long-time behavior, we can simply replace $t$ by infinity and the range of integration in eq. (3.5) is set to be $(-\infty, \infty)$. By expressing the Green's functions $G^{>}$and $G^{W}$ in terms of the spectral function $\rho$ [11]

$$
\begin{aligned}
G^{>}(\omega, \beta) & =\rho(\omega) \frac{1}{1+e^{-\beta \omega}}, \\
G^{W}(\omega, \beta) & =\rho(\omega) \frac{1}{2 \cosh (\beta \omega / 2)},
\end{aligned}
$$

and making use of the fact that $\rho(\omega)$ is even in $\omega$, we obtain the following expression:

$$
\frac{d S_{\mathrm{S}}^{(2)}}{d t}=2 V^{2} N \int_{0}^{\infty}\left[\frac{d \omega}{2 \pi} \frac{\rho_{\mathrm{S}}\left(\omega, 2 \beta_{S}\right) \rho_{\mathrm{E}}\left(\omega, \beta_{\mathrm{E}}\right)}{2 \cosh \beta_{S} \omega} \times \frac{\left(e^{\beta_{S} \omega}-1\right)\left(1-e^{\left(\beta_{\mathrm{E}}-\beta_{S}\right) \omega}\right)}{1+e^{\beta_{\mathrm{E}} \omega}}\right] .
$$

Note that in eq. (4.3), all terms are positive definite except for the $1-e^{\left(\beta_{\mathrm{E}}-\beta_{S}\right) \omega}$ term. When the temperature of the environment is lower than the temperature of the system, 
$\beta_{\mathrm{E}}>\beta_{\mathrm{S}}$ and $1-e^{\left(\beta_{\mathrm{E}}-\beta_{S}\right) \omega}<0$. Therefore, $d S_{\mathrm{S}}^{(2)} / d t<0$ and the entropy decreases at long time, which yields the Page curve. This gives a sufficient condition for the emergence of the Page curve, that is, $\kappa$ being small and the environment temperature lower than system temperature, which also agrees with the three aforementioned conditions.

The second understanding replies on inspecting how the system entropy saturates at sufficiently long times. It is reasonable to assume that the system eventually reaches thermal equilibrium with the environment, and since the environment contains much more degrees-of-freedom, the saturation entropy is determined by $\kappa$ and $\beta_{\mathrm{E}}$ and is independent of $\beta_{\mathrm{S}}$. The saturation entropy is smaller when the environment temperature is lower, which corresponds to a decrease in thermal entropy. When the coupling $\kappa$ is smaller the saturation entropy is also smaller because this lowers the entanglement entropy. On the other hand, the initial entropy of the system is solely determined by parameters $J_{\mathrm{S}}$ and $\beta_{\mathrm{S}}$ of the system itself. When this saturation entropy is smaller than the initial entropy, the entropy has to decrease at a later stage, which also leads to a sufficient condition for the emergence of the Page curve.

\section{CFT bath and holography}

Now we discuss possible gravity interpretation of our results. It is known that the lowenergy sector of the $\mathrm{SYK}_{4}$ model is described by a Schwarzian action [11], which is equivalent to the $\mathrm{AdS}_{2}$ Jackiw-Teitelboim gravity [32]. Consequently, for $\beta_{S} J_{S} \gg 1$, the initial thermal ensemble of the SYK system can be viewed as a Rindler patch of the $\mathrm{AdS}_{2}$ spacetime with gravity dynamics lying on the boundary. On the gravity side, previous works [6-9] usually introduce the bath as a (1+1)-D boundary conformal field theory (bCFT), which is glued to the $\mathrm{AdS}_{2}$ space-time with a transparent boundary condition.

In our model (2.1), we have chosen an $\mathrm{SYK}_{2}$ bath for simplicity, which is $0+1$-D. However, it is known that the $\mathrm{SYK}_{2}$ model shares a similar behavior to a higher-dimensinoal Fermi liquid [11]. In particular, we can introduce an alternative bath as in [26]:

$$
\hat{H}^{\prime}=\sum_{i<j<k<l} J_{i j k l}^{S} \psi_{i} \psi_{j} \psi_{k} \psi_{l}+\sum_{i}\left(\Lambda \sum_{x \geq 0} i \chi_{i, x} \chi_{i, x+1}+i V \psi_{i} \chi_{i, 0}\right) .
$$

Now the bath $\chi_{i, x}$ becomes $N$ copies of half-infinite Majorana chains, with $x=0,1,2 \ldots$ labeling different sites with lattice constant $a$. Although (5.1) and (2.1) describe very different microscopic systems, after integrating out the bath $\chi$, we find that the effective action of the system takes the same form (see the first and the third line of (A.8)), and only depends on the bath two-point function. Taking the limit of $\Lambda / J \rightarrow \infty$ with fixed $\Lambda a=2 \pi$, we find that in the new model we have $\rho_{\mathrm{E}}\left(\omega, \beta_{\mathrm{E}}\right) \sim 1 / \Lambda$, which is also a constant. Consequently, the Majorana chain bath model (5.1) is equivalent to the $\mathrm{SYK}_{2}$ bath model with $\Lambda=J_{E}$ and we can identify $\kappa=V^{2} / \Lambda$.

The holographic interpretation requires that the system can be described purely by the reparametrization modes in the low-energy manifold of the $\mathrm{SYK}_{4}$ model. This is valid in the regime $\kappa \sqrt{\beta_{S} / J_{S}} \ll \alpha_{S} / \beta_{S} J_{S}$ or $\kappa \beta_{S}^{3 / 2} J_{S}^{1 / 2} \ll 1[22], \alpha_{S}$ being the coefficient of the 
(a)

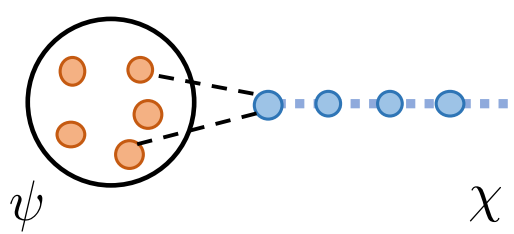

(b)

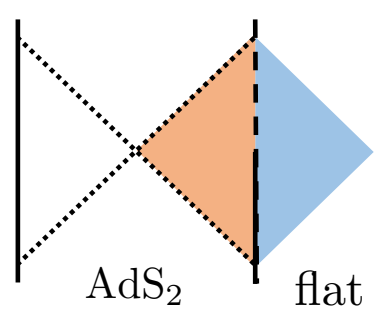

Figure 4. A sketch for (a). the chain model (5.1), and (b) its gravity dual. Here the orange color labels the system, which is an $\mathrm{SYK}_{4}$ model or a $\mathrm{AdS}_{2}$ region with gravity dynamics. The blue color labels the bath, described by a Majorana chain or CFT on a flat space-time.

Schwarzian action in the SYK model [11]. Since we expect the system to ultimately thermalize, $\beta_{S} J_{S}$ will eventually become small enough to spoil the holographic interpretation if the bath is initially prepared at a high temperature. Nevertheless, this can be avoided by either considering a low-temperature bath or focusing on the short time physics. Under these conditions, (5.1) then admits a direct gravity interpretation. The bulk system contains two regions (see figure 4), one with dynamical gravity, and one with flat space-time. This is similar to the setup in previous works [6-9]. The main difference is that our model contains no CFT degrees of freedom in the gravity region: using the holographic dictionary, the Majorana fermion with scaling dimension $\Delta=1 / 4$ is mapped to a two-component Majorana fermion satisfying the Dirac equation with mass $m=1 / 2-\Delta=1 / 4$. Fortunately, previous studies find this difference does not change the qualitative behavior of the entropy dynamics [26]. Our work reveals the effect of gluing AdS2 to the flat space-time beyond Markovian approximation. We find only in the short time limit, the effect of the flat spacetime region can be approximated by the Lindblad master equation. During this short period, the entanglement builds up due to free excitations passing through the boundary between the AdS2 and the flat space-time, long before the thermalization takes place. The Page curve only appears at later time when the Markovian approximation breaks down and the temperature of the bath is low enough. This is consistent with previous studies for CFT matters in bulk $[7,8]$. However, in these calculations, the entropy curve for Von Neumann entropy contains a transition for the RT surfaces, which is smoothed in our case. Whether having transitions or crossovers in the entropy dynamics in fact depends on the details of the system-bath coupling, as was noted in [26].

\section{Summary}

To summarize, we address the issue of when a Page curve can emerge in entropy dynamics of a system coupled to the environment. Although we use SYK model as an exactly solvable model to study this problem, the lesson we learn from our model reveals a general physical picture that should be applicable to generic chaotic quantum many-body systems. This physical picture contains two ingredients. First, at the initial stage, the entropy dynamics is always dominated by the Markovian process which leads to a linear increase of entropy in time. Secondly, a chaotic system thermalizes with the environment in the 
long-time limit. After thermalization, a low environment temperature and a weak systemenvironment coupling respectively suppress the thermal and the entanglement contributions to the system entropy, which ensures a lower system entropy at long time and forces the entropy to decrease at the later stage. The long-time decreasing behavior is essential for the emergence of the Page curve. This long-time behavior is distinct from the Markovian case where the system is often heated to infinite temperature and the long-time steady state is described by a density matrix given by the identity matrix. Therefore, the Page curve is a consequence of the non-Markovian environment. Since the entanglement entropy can now be measured experimentally [40-42] and the coupling to the environment can be also highly controllable, for instance, in ultracold atomic systems, the physical picture revealed in this work can be experimentally verified in near future.

\section{Acknowledgments}

We thank Yiming Chen, Yingfei Gu, and Zhenbin Yang for helpful discussion. This work is supported by Beijing Outstanding Young Scientist Program, NSFC Grant No. 11734010, MOST under Grant No. 2016YFA0301600.

Note added. When finishing the manuscript, we become aware of a work by Chen in which the Rényi entropy dynamics has been stuided in a similar model by the perturbation theory [43].

\section{A Path-integral representation of the Rényi entropy}

The method we use to calculate the entropy curve is the path-integral formalism [22, 26-31]. Here we briefly illustrate how this method can be tailored to solve our models.

\section{A.1 Non-Markovian bath}

We first write the initial thermal ensemble of the decoupled $\psi / \chi$ system with the inverse temperature $\beta_{S} / \beta_{B}$ as path-integrals. The initial density matrix is

$$
\rho(t=0)=\frac{1}{Z_{B} Z_{S}} e^{-\beta_{S} H_{S}} \otimes e^{-\beta_{B} H_{B}}=\frac{1}{Z_{B} Z_{S}}
$$

Here the solid arc represents the path integral on the imaginary time contour and the end points represent the bra or the ket. We then evolve the system $\rho$ under the total Hamiltonian eq. (2.1) in the main text, and obtain the reduced density matrix of $\psi$ by tracing out the bath $\chi$. This procedure is represented by the contour below:

$$
\rho_{S}(t)=\frac{1}{Z_{B} Z_{S}} \operatorname{tr}_{B}\left[e^{-i H t}\left(e^{-\beta_{S} H_{S}} \otimes e^{-\beta_{B} H_{B}}\right) e^{i H t}\right]=\frac{1}{Z_{B} Z_{S}}
$$


Here the dotted lines indicate the interaction between the system $\chi$ and the bath $\psi$. The $n$-th Rényi entropy is defined as

$$
S_{S}^{(n)}(t) \equiv-\frac{1}{n-1} \log \operatorname{tr}_{S}\left[\rho_{S}(t)^{n}\right] .
$$

For $n \rightarrow 1$, this gives the von Neumann entropy. As in the main text, we focus on the $n=2$ case. The purity $e^{-S_{S}^{(2)}(t)}=\operatorname{tr}_{S}\left[\rho_{S}(t)^{2}\right]$ can then be computed as path integral over the following contour

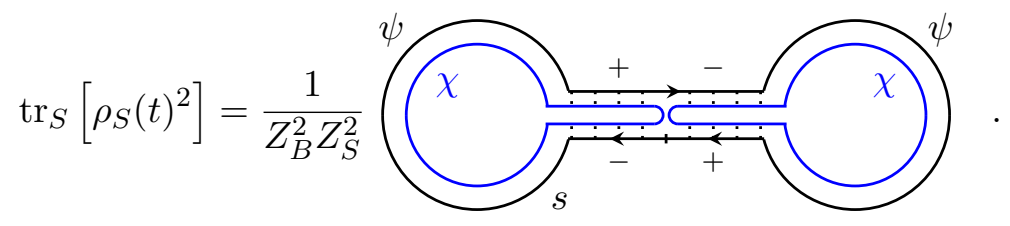

Here \pm represents the forward/backward unitary evolution. To proceed, we need to perform the disorder average over the random variables $\overline{S_{S}^{(2)}(t)}$. A strict treatment requires introducing additional disorder replicas. Here we assume that, to the leading order of $1 / N$, the disorder replicas are diagonal and we could interchange the order of performing the disorder average:

$$
\overline{S_{S}^{(2)}(t)}=-\overline{\log \operatorname{tr}_{S}\left[\rho_{S}(t)^{2}\right]} \approx-\log \operatorname{tr}_{S} \overline{\left[\rho_{S}(t)^{2}\right]} .
$$

We further introduce the bi-local fields following the standard procedure. We define

$$
G_{\psi}\left(s, s^{\prime}\right)=\frac{1}{N} \sum_{i} \psi_{i}(s) \psi_{i}\left(s^{\prime}\right), \quad G_{\chi}\left(l, l^{\prime}\right)=\frac{1}{M} \sum_{a} \chi_{a}(l) \chi_{a}\left(l^{\prime}\right) .
$$

Here $s \in\left[0,4 t+2 \beta_{S}\right)$ parametrizes the contour of $\psi$ (clockwise, as indicated in (A.4)) and similarly $l \in\left[0,4 t+2 \beta_{B}\right)$ parametrizes the contour of $\chi$. On the real-time evolution contour, the dotted lines induce a mapping from $s$ to $l(s)$. Further introducing the Lagrangian multiplier $\Sigma_{\psi / \chi}$ and integrating out the fermion fields, we find

$$
Z_{S}^{2} Z_{B}^{2} \operatorname{tr}_{S} \overline{\left[\rho_{S}(t)^{2}\right]}=\int \mathcal{D} G_{\psi} \mathcal{D} \Sigma_{\psi} \mathcal{D} G_{\chi} \mathcal{D} \Sigma_{\chi} \exp \left(-S_{\text {eff }}^{(2)}\right)
$$

where the action $S_{\text {eff }}^{(2)}$ is given by

$$
\begin{aligned}
S_{\mathrm{eff}}^{(2)}= & -\frac{N}{2} \log \left[\operatorname{det}_{\mathrm{S}}\left(\partial_{s}-\Sigma_{\psi}\right)\right]+\frac{N}{2} \int d s d s^{\prime}\left(\Sigma_{\psi} G_{\psi}-f(s) f\left(s^{\prime}\right) \frac{J_{S}^{2}}{4} G_{\psi}^{4}\right) \\
& -\frac{M}{2} \log \left[\operatorname{det}_{\mathrm{B}}\left(\partial_{l}-\Sigma_{\chi}\right)\right]+\frac{M}{2} \int d l d l^{\prime}\left(\Sigma_{\chi} G_{\chi}-g(l) g\left(l^{\prime}\right) \frac{J_{B}^{2}}{2} G_{\chi}^{2}\right) \\
& -\frac{N}{2} \int d s d s^{\prime} V^{2} p(s) p\left(s^{\prime}\right) G_{\psi}\left(s, s^{\prime}\right) G_{\chi}\left(l(s), l\left(s^{\prime}\right)\right) .
\end{aligned}
$$

Here $S / B$ under the "det" indicates different boundary condition for the system and the bath:

$$
\psi\left(0^{+}\right)=-\psi\left(2 \beta_{S}+4 t^{-}\right), \quad \chi\left(0^{+}\right)=-\chi\left(\beta_{B}+2 t^{-}\right), \quad \chi\left(\beta_{B}+2 t^{+}\right)=-\chi\left(2 \beta_{B}+4 t^{-}\right) .
$$


$f(s), g(l)$ and $p(s)$ are introduced to distinguish the forward or the backward real-time or imaginary-time evolutions. We have $f(s)=1, g(l)=1$ and $p(s)=0$ if $s$ or $l$ lies on the imaginary-time evolution and $f(s)= \pm i, g(l)= \pm i$ and $p(s)= \pm i$ if $s$ or $l$ lies on the forward/backward real-time evolution. Explicitly, for $s$ we have

$$
\begin{array}{ll}
\text { imaginary } & s \in\left[t, t+\beta_{S}\right] \cup\left[3 t+\beta_{S}, 3 t+2 \beta_{S}\right], \\
\text { forward } & s \in\left[t+\beta_{S}, 2 t+\beta_{S}\right] \cup\left[3 t+2 \beta_{S}, 4 t+2 \beta_{S}\right], \\
\text { backward } & s \in[0, t] \cup\left[2 t+\beta_{S}, 3 t+\beta_{S}\right] .
\end{array}
$$

For $l$, we just need to replace $\beta_{S}$ by $\beta_{B}$. In the large- $N$ limit, we could take the saddle-point equation. For system $\psi$, we have

$$
\begin{aligned}
& G_{\psi}\left(s, s^{\prime}\right)=\left(\partial_{s}-\Sigma_{\psi}\right)_{S}^{-1}\left(s, s^{\prime}\right), \\
& \Sigma_{\psi}\left(s, s^{\prime}\right)=J_{S}^{2} G_{\psi}^{3}\left(s, s^{\prime}\right) f(s) f\left(s^{\prime}\right)+V^{2} G_{\chi}\left(l(s), l\left(s^{\prime}\right)\right) p(s) p\left(s^{\prime}\right),
\end{aligned}
$$

and for the bath $\chi$, we have

$$
G_{\chi}\left(l, l^{\prime}\right)=\left(\partial_{l}-\Sigma_{\chi}\right)_{B}^{-1}\left(l, l^{\prime}\right), \quad \Sigma_{\chi}\left(l, l^{\prime}\right)=J_{B}^{2} G_{\chi}\left(l, l^{\prime}\right) g(l) g\left(l^{\prime}\right) .
$$

Note that the Schwinger-Dyson equation for the bath $\chi$ does not contain the Green's function of $\psi$, further indicating that the bath is unaffected by the system. Consequently, the Green's function of $\chi$ is diagonal in the two replica space and takes the form of the Green's functions on the Keldysh contour.

After solving (A.11), we can compute the on-shell action with eq. (A.8) and obtain the second Rényi entropy

$$
\overline{S_{S}^{(2)}(t)}=\frac{N}{2} \log \left[\operatorname{det}_{\mathrm{S}} G_{\psi}\right]+\frac{N}{2} \int d s d s^{\prime} f(s) f\left(s^{\prime}\right) \frac{3 J_{S}^{2}}{4} G_{\psi}^{4}+2 \log Z_{S}
$$

Here we have used the fact that the contribution from $\chi$ cancels $2 \log Z_{B}$.

\section{A.2 Markovian bath}

Similarly, we can consider the evolution described by the master equation eq. (3.1) in the main text. Now the contour can be drawn as

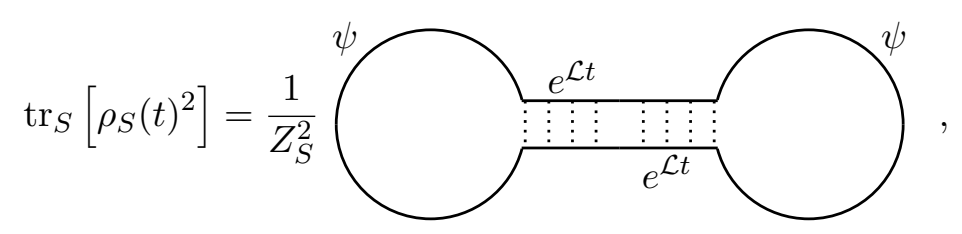

where the dotted lines now represent the insertion of the Lindblad operators. Again, we can relate the purity to a path-integral and introduce bi-local fields. We then arrive at

$$
Z_{S}^{2} \operatorname{tr}_{S} \overline{\left[\rho_{S}(t)^{2}\right]}=\int \mathcal{D} G_{\psi} \mathcal{D} \Sigma_{\psi} \exp \left(-S_{\text {eff }}^{(2)}\right)
$$


with the action $S_{\text {eff }}^{(2)}$

$$
\begin{aligned}
S_{\mathrm{eff}}^{(2)}= & -\frac{N}{2} \log \left[\operatorname{det}\left(\partial_{s}-\Sigma_{\psi}\right)\right]+\frac{N}{2} \int d s d s^{\prime}\left(\Sigma_{\psi} G_{\psi}-f(s) f\left(s^{\prime}\right) \frac{J_{S}^{2}}{4} G_{\psi}^{4}\right) \\
& -\frac{N \kappa}{2} \int d s d s^{\prime} G_{\psi}\left(s, s^{\prime}\right) g\left(s, s^{\prime}\right)+N \kappa t .
\end{aligned}
$$

Here $g\left(s, s^{\prime}\right)=-g\left(s^{\prime}, s\right)$ is an anti-symmetric function. For $s>s^{\prime}$, it is non-zero when

$$
\begin{array}{ll}
g\left(s, s^{\prime}\right)=\delta\left(\beta+2 t-s-s^{\prime}\right) & \text { if } s^{\prime} \in[0, t], \\
g\left(s, s^{\prime}\right)=\delta\left(3 \beta+6 t-s-s^{\prime}\right) & \text { if } s^{\prime} \in\left[\beta_{S}+2 t, \beta_{S}+3 t\right] .
\end{array}
$$

The $\psi$ system again satisfies the boundary condition $\psi\left(0^{+}\right)=-\psi\left(2 \beta_{S}+4 t^{-}\right)$when we compute the entropy. The saddle-point equation now reads

$$
\begin{aligned}
& G_{\psi}\left(s, s^{\prime}\right)=\left(\partial_{s}-\Sigma_{\psi}\right)_{S}^{-1}\left(s, s^{\prime}\right), \\
& \Sigma_{\psi}\left(s, s^{\prime}\right)=J_{S}^{2} G_{\psi}^{3}\left(s, s^{\prime}\right) f(s) f\left(s^{\prime}\right)+\kappa g\left(s, s^{\prime}\right) .
\end{aligned}
$$

The second Rényi entropy $\overline{S_{S}^{(2)}(t)}$ can again be calculated similar to eq. (A.13) as:

$$
\overline{S_{S}^{(2)}(t)}=\frac{N}{2} \log \left[\operatorname{det}_{\mathrm{S}} G_{\psi}\right]+\frac{N}{2} \int d s d s^{\prime} f(s) f\left(s^{\prime}\right) \frac{3 J_{S}^{2}}{4} G_{\psi}^{4}+N \kappa t+2 \log Z_{S} .
$$

\section{B Perturbation results}

In this section, we elaborate on details of the perturbation theory introduced in the main text. The parameter that controls the perturbation is $\kappa t$, so our results apply both for small $\kappa$ and short $t$. Below we illustrate these two aspects respectively.

\section{B.1 The short-time perturbation}

We first consider the case where $t$ is small and illustrate our perturbation scheme. This result is crucial for understanding the universal initial growth in the main text. At $t=0$, the system is in a thermal ensemble, and $\overline{S_{S}^{(2)}(0)}$ is given by the thermal entropy with the inverse temperature $\beta_{S}$. When the real-time evolution begins, the system entangles with the bath, and the entropy grows. In this early-time regime, we could treat the coupling to bath perturbatively.

We firstly consider the Markovian case where the early-time result can be computed analytically. To the leading order of $\kappa$, we need to evaluate the second line of eq. (A.16) directly using the Green's function at $\kappa=0$. We then have:

$$
\overline{S_{S}^{(2)}(t)} \approx \overline{S_{S}^{(2)}(0)}+N \kappa t\left(1-2 G_{\psi}^{W}\left(0,2 \beta_{S}\right)\right) .
$$

This formula works for $\kappa t \lesssim 1$. Here we have defined the Wightman Green's function

$$
G_{\psi}^{W}(t, \beta)=\frac{1}{Z(\beta)}\left\langle\psi_{i}\left(t-i \frac{\beta}{2}\right) \psi_{i}(0)\right\rangle_{\beta}
$$


for a single $\mathrm{SYK}_{4}$ model. In the low-energy limit $\beta_{S} J_{S} \gg 1$, we have $G_{\psi}^{W}(0, \beta)=\left(\frac{\pi}{4 J_{S}^{2} \beta^{2}}\right)^{1 / 4}$ and

$$
\overline{S_{S}^{(2)}(t)} \approx \overline{S_{S}^{(2)}(0)}+N \kappa t\left[1-\left(\frac{\pi}{J_{S}^{2} \beta^{2}}\right)^{1 / 4}\right] .
$$

We now consider the $\mathrm{SYK}_{2}$ bath. We similarly perform a perturbative calculation in terms of $V^{2}$. We then need to compute

$$
\overline{S_{S}^{(2)}(t)} \approx \overline{S_{S}^{(2)}(0)}-\frac{N}{2} \int d s d s^{\prime} V^{2} p(s) p\left(s^{\prime}\right) G_{\psi}\left(s, s^{\prime}\right) G_{\chi}\left(l(s), l\left(s^{\prime}\right)\right)
$$

using the Green's function with $V=0$. Instead of using the parameter $s$, we could now directly focus on a single replica and write the integral in terms of the real-time $t$ :

$$
2 V^{2} N \int_{0}^{t} \int_{0}^{t} d t_{1} d t_{2}\left[G_{\psi}^{T}\left(t_{12}, 2 \beta_{S}\right) G_{\chi}^{T}\left(t_{12}, \beta_{B}\right)-G_{\psi}^{W}\left(t_{12}, 2 \beta_{S}\right) G_{\chi}^{>}\left(t_{12}, \beta_{B}\right)\right] .
$$

Here $G^{T}$ is the real-time time-ordered Green's function and $G_{\chi}^{>}\left(t, \beta_{B}\right)=\left\langle\chi_{i}(t) \chi_{i}(0)\right\rangle_{\beta_{B}}$. On the other hand, the perturbation of the partition function should be strictly zero. Then, it yields an equality as

$$
2 V^{2} N \int_{0}^{t} \int_{0}^{t} d t_{1} d t_{2}\left[G_{\psi}^{T}\left(t_{12}, \beta_{S}\right) G_{\chi}^{T}\left(t_{12}, \beta_{B}\right)-G_{\psi}^{>}\left(t_{12}, \beta_{S}\right) G_{\chi}^{>}\left(t_{12}, \beta_{B}\right)\right]=0
$$

Substituting this equality into eq. (B.4) we obtain

$$
2 V^{2} N \int_{0}^{t} \int_{0}^{t} d t_{1} d t_{2}\left[G_{\psi}^{>}\left(t_{12}, 2 \beta_{S}\right) G_{\chi}^{>}\left(t_{12}, \beta_{B}\right)-G_{\psi}^{W}\left(t_{12}, 2 \beta_{S}\right) G_{\chi}^{>}\left(t_{12}, \beta_{B}\right)\right]
$$

We now focus on the initial time behavior of this perturbation term. Specifically, we take its derivative with respect to $t$, which gives

$$
\frac{d \Delta S_{S}^{(2)}(t)}{d t}=2 V^{2} N \int_{-t}^{t} d t_{12}\left(G_{\psi}^{>}\left(t_{12}, 2 \beta_{S}\right)-G_{\psi}^{W}\left(t_{12}, 2 \beta_{S}\right)\right) G_{\chi}^{>}\left(t_{12}, \beta_{B}\right)
$$

\section{B.2 Long time asymptotic behavior with small $\kappa$}

The small $\kappa$ perturbation theory is utilized to illustrate the emergence of the page curves. Revisiting (B.7) we note that there are two limits where we can obtain analytical results. The first limit is when $t \rightarrow 0$, which gives the initial entropy growth. The second limit is when $t \rightarrow \infty$, revealing the asymptotic entropy growth rate. We find that the sign of the asymptotic growth rate only depends on whether the bath temperature is higher or lower than the system temperature. To find the asymptotic growth rate we simply take $t$ to infinity in eq. (B.7). We now show that this expression can be cast into a form in which the temperature dependence is manifest. 
Writing the Green's functions in terms of the spectral function with the help of the fluctuation-dissipation theorem, we obtain the following expression

$$
\begin{aligned}
R & =2 V^{2} N \int_{-\infty}^{\infty} d t_{12}\left(G_{\psi}^{>}\left(t_{12}, 2 \beta_{S}\right)-G_{\psi}^{W}\left(t_{12}, 2 \beta_{S}\right)\right) G_{\chi}^{>}\left(t_{12}, \beta_{B}\right) \\
& =2 V^{2} N \int_{-\infty}^{\infty} \frac{d \omega}{2 \pi} G_{\chi}^{>}\left(\omega, \beta_{B}\right)\left(G_{\psi}^{>}\left(-\omega, 2 \beta_{S}\right)-G_{\psi}^{W}\left(-\omega, 2 \beta_{S}\right)\right) \\
& =2 V^{2} N \int_{-\infty}^{\infty} \frac{d \omega}{2 \pi} \frac{\rho_{\psi}\left(\omega, 2 \beta_{S}\right) \rho_{\chi}\left(\omega, \beta_{B}\right)}{2 \cosh \beta_{S} \omega}\left(\frac{e^{-\beta_{S} \omega}-1}{1+e^{-\beta_{B} \omega}}+\frac{e^{\beta_{S} \omega}-1}{1+e^{\beta_{B} \omega}}\right) \times \frac{1}{2} \\
& =2 V^{2} N \int_{0}^{\infty} \frac{d \omega}{2 \pi} \frac{\rho_{\psi}\left(\omega, 2 \beta_{S}\right) \rho_{\chi}\left(\omega, \beta_{B}\right)}{2 \cosh \beta_{S} \omega} \frac{\left(e^{\beta_{S} \omega}-1\right)\left(1-e^{\left(\beta_{B}-\beta_{S}\right) \omega}\right)}{1+e^{\beta_{B} \omega}} .
\end{aligned}
$$

From this expression we can easily see that the sign of the long time derivative is solely determined by $\beta_{B}-\beta_{S}$. When the bath temperature is lower than that of the system, the perturbation term decreases linearly in the long time limit. This result is general in the sense that it only requires the coupling between the system and the bath to be linear. The bath can in principle be any SYK-like bath and this would still apply. We leave more complex coupling terms, such as those involving higher order terms, for future discussions.

\section{Numerical discretization}

Equations (A.11) and (A.18) solely determine the Rényi entropy of our system in the large$N$ limit at any given $t$. It is however difficult to study them analytically since we do not have time-translational symmetry. Instead, we resort to a numerical study, the details of which will be described below [26-30]. We illustrate it with eq. (A.18) as an example.

We will use an iterative approach to solve eq. (A.18). The contour variable $\mathrm{s} \in[0,2 \beta+4 t)$ can be discretized with interval $\beta / L$, then both $G_{\psi}$ and $\Sigma_{\psi}$ become $\left(2+4 t^{\prime}\right) L \times\left(2+4 t^{\prime}\right) L$ matrices, where $t^{\prime}=t / \beta$. We typically take $L=80 \sim 160$. After the discretization, the Schwinger-Dyson equation is written as:

$$
\begin{aligned}
& \left(G_{\psi}\right)_{i j}=\left(\left(G_{\psi}^{0}\right)^{-1}-\Sigma_{\psi}\right)_{i j}^{-1}, \\
& \left(\Sigma_{\psi}\right)_{i j}=J_{S}^{2}\left(G_{\psi}\right)_{i j}^{3} f_{i} f_{j}+V^{2}\left(G_{\chi}\right)_{i j} p_{i} p_{j} .
\end{aligned}
$$

To obtain $\left(G_{\psi}\right)_{i j}^{-1}$ we need to find the matrix of $\left(G_{\psi}\right)_{i j}$. Taking into account the boundary condition for the bare Green's function, we have:

$$
\left(G_{\psi}^{0}\right)_{i j}=\frac{1}{2} \operatorname{sgn}(i-j), \quad \text { for } \quad i, j \in\left\{1,2, \ldots\left(2 \beta+4 t^{\prime}\right) L\right\} .
$$

After the iteration converges, we can plug the solution matrix back into the action and then obtain the entropy value. To further solidify our results we have performed an extrapolation for different $L$.

Open Access. This article is distributed under the terms of the Creative Commons Attribution License (CC-BY 4.0), which permits any use, distribution and reproduction in any medium, provided the original author(s) and source are credited. 


\section{References}

[1] J. Preskill, Quantum computing in the NISQ era and beyond, Quantum 2 (2018) 79.

[2] M.O. Scully and M.S. Zubairy, Quantum Optics, Cambridge University Press, Cambridge U.K. (1997).

[3] H.P. Breuer and F. Petruccione, The Theory of Open Quantum Systems, Oxford University Press, Oxford U.K. (2007).

[4] S.W. Hawking, Particle Creation by Black Holes, Commun. Math. Phys. 43 (1975) 199 [Erratum ibid. 46 (1976) 206] [INSPIRE].

[5] D.N. Page, Average entropy of a subsystem, Phys. Rev. Lett. 71 (1993) 1291 [gr-qc/9305007] [INSPIRE].

[6] G. Penington, Entanglement Wedge Reconstruction and the Information Paradox, JHEP 09 (2020) 002 [arXiv: 1905.08255] [InSPIRE].

[7] A. Almheiri, N. Engelhardt, D. Marolf and H. Maxfield, The entropy of bulk quantum fields and the entanglement wedge of an evaporating black hole, JHEP 12 (2019) 063 [arXiv: 1905.08762] [INSPIRE].

[8] A. Almheiri, R. Mahajan, J. Maldacena and Y. Zhao, The Page curve of Hawking radiation from semiclassical geometry, JHEP 03 (2020) 149 [arXiv: 1908.10996] [INSPIRE].

[9] A. Almheiri, T. Hartman, J. Maldacena, E. Shaghoulian and A. Tajdini, Replica Wormholes and the Entropy of Hawking Radiation, JHEP 05 (2020) 013 [arXiv:1911.12333] [INSPIRE].

[10] G. Penington, S.H. Shenker, D. Stanford and Z. Yang, Replica wormholes and the black hole interior, arXiv: 1911.11977 [INSPIRE].

[11] J. Maldacena and D. Stanford, Remarks on the Sachdev-Ye-Kitaev model, Phys. Rev. D 94 (2016) 106002 [arXiv:1604.07818] [INSPIRE].

[12] A. Kitaev and S.J. Suh, The soft mode in the Sachdev-Ye-Kitaev model and its gravity dual, JHEP 05 (2018) 183 [arXiv:1711.08467] [INSPIRE].

[13] Y. Gu, X.-L. Qi and D. Stanford, Local criticality, diffusion and chaos in generalized Sachdev-Ye-Kitaev models, JHEP 05 (2017) 125 [arXiv: 1609.07832] [INSPIRE].

[14] R.A. Davison, W. Fu, A. Georges, Y. Gu, K. Jensen and S. Sachdev, Thermoelectric transport in disordered metals without quasiparticles: The Sachdev-Ye-Kitaev models and holography, Phys. Rev. B 95 (2017) 155131 [arXiv:1612.00849] [INSPIRE].

[15] X. Chen, R. Fan, Y. Chen, H. Zhai and P. Zhang, Competition between Chaotic and Nonchaotic Phases in a Quadratically Coupled Sachdev-Ye-Kitaev Model, Phys. Rev. Lett. 119 (2017) 207603 [arXiv:1705.03406] [INSPIRE].

[16] S. Banerjee and E. Altman, Solvable model for a dynamical quantum phase transition from fast to slow scrambling, Phys. Rev. B 95 (2017) 134302 [arXiv:1610.04619] [InSPIRE].

[17] S.-K. Jian and H. Yao, Solvable Sachdev-Ye-Kitaev models in higher dimensions: from diffusion to many-body localization, Phys. Rev. Lett. 119 (2017) 206602 [arXiv:1703.02051] [INSPIRE].

[18] X.-Y. Song, C.-M. Jian and L. Balents, Strongly Correlated Metal Built from Sachdev-Ye-Kitaev Models, Phys. Rev. Lett. 119 (2017) 216601 [arXiv:1705.00117] [INSPIRE]. 
[19] P. Zhang, Dispersive Sachdev-Ye-Kitaev model: Band structure and quantum chaos, Phys. Rev. B 96 (2017) 205138 [arXiv: 1707. 09589] [INSPIRE].

[20] C.-M. Jian, Z. Bi and C. Xu, A model for continuous thermal Metal to Insulator Transition, Phys. Rev. B 96 (2017) 115122 [arXiv:1703.07793] [inSPIRE].

[21] A. Eberlein, V. Kasper, S. Sachdev and J. Steinberg, Quantum quench of the Sachdev-Ye-Kitaev Model, Phys. Rev. B 96 (2017) 205123 [arXiv:1706. 07803] [InSPIRE].

[22] Y. Gu, A. Lucas and X.-L. Qi, Spread of entanglement in a Sachdev-Ye-Kitaev chain, JHEP 09 (2017) 120 [arXiv: 1708.00871] [INSPIRE].

[23] Y. Chen, H. Zhai and P. Zhang, Tunable quantum chaos in the Sachdev-Ye-Kitaev model coupled to a thermal bath, JHEP 07 (2017) 150 [arXiv:1705.09818] [INSPIRE].

[24] P. Zhang, Evaporation dynamics of the Sachdev-Ye-Kitaev model, Phys. Rev. B 100 (2019) 245104 [arXiv: 1909.10637] [INSPIRE].

[25] A. Almheiri, A. Milekhin and B. Swingle, Universal Constraints on Energy Flow and SYK Thermalization, arXiv:1912.04912 [INSPIRE].

[26] Y. Chen, X.-L. Qi and P. Zhang, Replica wormhole and information retrieval in the SYK model coupled to Majorana chains, JHEP 06 (2020) 121 [arXiv:2003.13147] [INSPIRE].

[27] P. Zhang, Entanglement Entropy and its Quench Dynamics for Pure States of the Sachdev-Ye-Kitaev model, JHEP 06 (2020) 143 [arXiv: 2004.05339] [INSPIRE].

[28] P. Zhang, C. Liu and X. Chen, Subsystem Rényi Entropy of Thermal Ensembles for SYK-like models, SciPost Phys. 8 (2020) 094 [arXiv: 2003.09766] [InSPIRE].

[29] C. Liu, P. Zhang and X. Chen, Non-unitary dynamics of Sachdev-Ye-Kitaev chain, SciPost Phys. 10 (2021) 048 [arXiv: 2008.11955] [INSPIRE].

[30] S.-K. Jian and B. Swingle, Note on entropy dynamics in the Brownian SYK model, arXiv: 2011.08158 [INSPIRE].

[31] P. Dadras and A. Kitaev, Perturbative calculations of entanglement entropy, JHEP 03 (2021) 198 [arXiv : 2011. 09622] [inSPIRE].

[32] J. Maldacena, D. Stanford and Z. Yang, Conformal symmetry and its breaking in two-dimensional nearly anti-de sitter space, PTEP 2016 (2016) 12C104.

[33] J. Polchinski and V. Rosenhaus, The Spectrum in the Sachdev-Ye-Kitaev Model, JHEP 04 (2016) 001 [arXiv: 1601.06768] [INSPIRE].

[34] K. Jensen, Chaos in AdS 2 Holography, Phys. Rev. Lett. 117 (2016) 111601 [arXiv: 1605.06098] [INSPIRE].

[35] A. Jevicki and K. Suzuki, Bi-Local Holography in the SYK Model: Perturbations, JHEP 11 (2016) 046 [arXiv: 1608.07567] [INSPIRE].

[36] G. Mandal, P. Nayak and S.R. Wadia, Coadjoint orbit action of Virasoro group and two-dimensional quantum gravity dual to SYK/tensor models, JHEP 11 (2017) 046 [arXiv: 1702.04266] [INSPIRE].

[37] D.J. Gross and V. Rosenhaus, The Bulk Dual of SYK: cubic couplings, JHEP 05 (2017) 092 [arXiv: 1702.08016] [INSPIRE].

[38] Y.-N. Zhou, L. Mao and H. Zhai, Renyi Entropy Dynamics and Lindblad Spectrum for Open Quantum System, arXiv:2101.11236 [INSPIRE]. 
[39] L. Pan, X. Chen, Y. Chen and H. Zhai, Non-hermitian linear response theory, Nature Phys. 16 (2020) 767.

[40] R. Islam et al., Measuring entanglement entropy in a quantum many-body system, Nature 528 (2015) 77.

[41] A.M. Kaufman et al., Quantum thermalization through entanglement in an isolated many-body system, Science 353 (2016) 794.

[42] A. Lukin et al., Probing entanglement in a many-body-localized system, Science 364 (2019) 256 [arXiv: 1805.09819].

[43] Y. Chen, Entropy linear response theory with non-Markovian bath, JHEP 04 (2021) 215 [arXiv: 2012.00223] [INSPIRE]. 